\title{
Visual Acuity Stability of Ortho-K Treatment in a Child with Low Myopia: A Case Study
}

\author{
Muliani \\ Anatomy Department of Medical Faculty of Udayana University, P. B. Sudirman Street, Denpasar, Bali 80232
}

\begin{abstract}
Myopia progressivity can be slowed by ortho-K lens (orthokeratology) which will flatten the cornea temporarily. This lens was safe and effective especially for children. Stability of visual acuity generally obtained between days 5 to 10. This case report is reporting about visual acuity stability of ortho-k treatment in a child with low myopia. An $11^{\text {th }}$ year old girl with low myopia was given fitted ortho-K lens. The results of visual acuity and corneal on day 2, 4, 7, 45, 180 and 187 were 6/6 OD as well as OS and flatten of the cornea. The eyes of the patient in this case have already adapted and stable. This is consistent with several other studies that stated orthokeratology can slow the progression of myopia by flattening the cornea and slows the axial elongation of the eye. Stability has occurred within 2 weeks after the first usage. It was concluded that orthokeratology is very effective and safe for children with myopia, especially at low degrees of myopia without astigmatism. Orthokeratology usage resulting in increased visual acuity and corneal flattening. Visual acuity stability has been reached in the first week. Orthokeratology in children with low myopia can be used every 2 days. Further research is needed to determine the effects of long term use of orthokeratology.
\end{abstract}

Keywords: orthokeratology, myopia, visual acuity, cornea, stability

\section{Introduction}

Advance in communication technology (smartphone, tablet, android), increase amount of social media and human social need, causing an increase of frequency and duration of eyes to look at a small screen in closer distance.

Myopia is one of eye refraction disorder. Shadows are formed at the anterior of the retina so it cannot see distant object clearly. This is due to axial elongation in myopia patient ${ }^{1}$. Increased prevalence of myopia in the world recently, especially in Southeast ${ }^{1,2}$ and East Asia (80-90\%) ${ }^{3}$ is a serious health problem mainly for patients with high myopia ${ }^{4}$. Myopia could occur as a result of heredity, ethnicity and environment, such as reading habit, often doing close activities ${ }^{5,6}$. Complication of high myopia is macula degeneration, glaucoma, cataract, strabismus and blindness ${ }^{1}$. This condition can decrease the quality of life in children and adult ${ }^{4}$. Progressivity of myopia can be slowed by using ortho-K lens (orthokeratology) ${ }^{7}$.

Orthokeratology is rigid contact lenses which reshape cornea into flat and reduce corneal eccentricity. These changes is temporary and do not repair the cause of myopia ${ }^{8}$. This technique is safe and effective especially for children to reduce myopia progressivity ${ }^{6,7}$. Often complication from this lens are: corneal staining (usually mild, can be monitored and easily handled) and microbial keratitis. This complication can be minimized by giving specific education to parents, children and doing routine eye examination ${ }^{6}$. Usage of this lens requires adaptation before gaining visual stability. Adaptation to this lens is needed to get visual acuity stability. Visual acuity stability usually gained between days 5 to 10 after wearing the lens ${ }^{9}$.

Monitoring should be done in the morning after first night using, third night, seventh night, second week and so on until the visual stability reached. After that, it should be checked routinely every three months ${ }^{9}$. This case report is reporting about visual acuity stability of ortho-k treatment in a child with low myopia.

\section{Case reports}

An $11^{\text {th }}$ year old girl came to eye specialist at Klinik Hidup Baru, Jakarta on June $24^{\text {th }}, 2015$ at 08.00 am. The girl has been wearing farsighted glasses since she was 8 . Twice a year, her parents always bring her to an optic to check her eyes. Every time she checked, the visual acuity always decreased. She was using -1.50 D (OD) dan -2.0 D, (OS) glasses when checked by orthokeratologist at the clinic.

Visual acuity and corneal topography were examined according to the procedure. Fit ortho-K lenses then given to the girl and used for one hour. After that, the lenses were removed then the visual acuity and corneal topography were reexamined. There was an increased in visual acuity. After being given informed consent, her parents decided to use ortho-K lens for the girl. The patient and her parents were taught the way to rinse the lens with Kery lens soap before and after using the lens, and then wash it with sterile water. After washed and will be used, put a drop of Kery reconditioning lens. The lenses were used only at night for 6 -8 hours and while being used, the patient should close her eyes. The lenses were removed in the next morning with a special pipette. Before being removed, each eye should have been given 5 drops of artificial tear (cendo litter) with interval 1 minute between each drop. One drop of cendo litter should be drip also on the pipette. After being removed, rinse the lenses with Kery lens soap, wash it, then put on the lens container and soaked with Kery lens soap.

After two days, the patient can see far things clearly without glasses. Visual acuity has been reached 6/6 OD and OS and the cornea has been flatten.

In two days later, the orthokeratologist found the same results in visual acuity and cornea as before which are 6/6 for both eyes and flatten of the cornea. 


\section{International Journal of Science and Research (IJSR) \\ ISSN (Online): 2319-7064}

Index Copernicus Value (2015): 78.96 | Impact Factor (2015): 6.391

Visual acuity and corneal topography were reexamined on day 7 and the results was 6/6 OD and OS while the cornea was flatten. According to anamnesis, the girl can still see clearly throughout the day. Due to the patient's domicile (out of town) so the control was scheduled 45 days later.

Visual acuity and corneal topography on day 45 was 6/6 OD as well as OS and the cornea was flatten. Patient can see far things clearly all day without any vision changes even when she was not used the lenses for one night. Results from examination on day 180 and 187 , were same as before, the visual acuity were $6 / 6 \mathrm{OD}$ as well as OS and flatten of the cornea.

\section{Discussions}

The eyes of the girl in this case, have been adapted and stable. This can be seen from the clear vision all day even when not wearing lenses for one night and the visual acuity were 6/6 OD and OS. This condition can occur due to the girl's age when first wearing the lens, short duration of low myopia so the cornea can return quickly to its original shape.

Research by Charm and Cho ${ }^{10}$ succeeded in decreasing myopia level in children with high myopia $(\geq 6.00 \mathrm{D})$ with average decrease about $3.75 \mathrm{D}$ on right eye. Visual acuity increased one month after wearing ortho-K lens without decreased of eye health.

Chen and $\mathrm{Cho}^{11}$ reported 2 children with high myopia and astigmatism which were given toric orthokeratology. After one year, myopia and astigmatism decreased in the two subjects. Result of the second subject is better than the first so myopia and astigmatism decrease much more. The etiology of this variation has not been known. Even though in all subjects there was no added in axial length.

According to case report which written by Baertschi and Wyss $^{12}$, the toric orthokeratology lens design FOKX can safely reduce high astigmatism and myopia.

Jung $^{8}$ concluded that many eye specialists in Korea are likely suggested myopia children to wear ortho-K lens than spectacles but not in long term.

Several factors that influence the orthokeratology effectiveness are: hyperopia at the peripheral retina, relative changes of the peripheral power of cornea, parental myopia level and pupil measurement. These make the orthokeratology mechanism in slow down myopia progression became more complex. One of the aims of the mechanism is to slow down the axial elongation so the myopia progressivity will be inhibits. Peripheral retinal hyperopia usually found in children with myopia. This hyperopia is the sign of the eye growth. Hyperopia can be decreased by ortho-K lens. This lens will cause flat of the central cornea meanwhile relatively steep the mid peripheral cornea and decrease myopia axial length so the peripheral cornea become more myopia. Peripheral myopia due to ortho-K lens will reduce peripheral hyperopic defocus and slow down the myopia progressivity ${ }^{13}$.
Cho et $\mathrm{al}^{14}$ did a research on 70 children between age $7-12^{\text {th }}$ years old whom is wearing spectacles. These children divided into 2 groups. The first group was given ortho-K lens meanwhile the second still worn spectacles. This research found that there is cornea flattening significantly on the first group although the effect on each child cannot be predicted due to variation on elongation axial length in children. There are also changes in axial length, vitreous chamber depth (VCD) and baseline spherical equivalent refractive errors (SER) after wore ortho-K lens for 2 years but the differences is not significant.

Chan et $\mathrm{al}^{15}$ have been doing research for 6 month, on 108 of 9 years old children with average myopia $-3,56 \pm 1,49 \mathrm{D}$ and cylinder $-0,50 \mathrm{D}$. This research found an increased in visual acuity without glasses or any other visual aid. The corneal shape was changed. The two changes were happened after using ortho-K lens on the first night. Stabilization happened in 2 weeks after first night using. Ortho-K lens under tight supervision is safe and effective for low to moderate myopia. Common problems usually found are corneal staining, lens binding and ocular discharge. Patients and their family were satisfied with the results from this research.

\section{Conclusion}

Orthokeratology is very effective and safe to use in myopia children especially low degree myopia without astigmatism. Orthokeratology usage resulting in increased visual acuity and corneal flattening. Visual acuity stability has been reached in the first week Orthokeratology in low myopia children can be used every 2 days. Further research is needed to know the effects of long term use of orthokeratology.

\section{Conflict of Interest}

None declared.

\section{Funding}

This research received no specific grant from any funding agency in the public, commercial, or not-for-profit sectors.

\section{References}

[1] Williams KM, Bertelsen G, Cumberland P, et al. Increasing Prevalence of Myopia in Europe and the Impact of Education. Ophthalmology 2015; 122(7): 1489-97.

[2] French AN, Morgan IG, Burlutsky G, et al. Prevalence and 5-to 6- Year Incidence and Progression of Myopia and Hyperopia in Australian Schoolchildren. Ophtalmology 2013; 120(7): 1482-91.

[3] Sun Y, Xu F, Zhang T, et al. Orthokeratology to Control Myopia Progression: A Meta Analysis. Journal PLos ONE. 2015; 10(4).

[4] Deborah J and Doerte L. The Prevalence and Impact of High Myopia. Eye \& Contact Lens: Science \& Clinical Practice 2012; 38(3): 188-196 


\section{International Journal of Science and Research (IJSR) \\ ISSN (Online): 2319-7064 \\ Index Copernicus Value (2015): 78.96 | Impact Factor (2015): 6.391}

[5] French AN, Morgan IG, Mitchell P, et al. Risk Factor for Incident Myopia in Australian Schoolchildren. Ophtalmology 2013; 120(10): 2100-08.

[6] Wen D, Huang J, Chen H, et al. Review Article: Efficacy and Acceptability of Orthokeratology for Slowing Myopic Progression in Children: A Systematic Review and Meta-Analysis. Journal of Ophthalmology 2015.

[7] Davis RL, Eiden SB, Bennett ES, et al. Stabilizing Myopia by Accelerating Reshaping Technique (SMART)-Study Three Year Outcomes and Overview. Adv Ophthalmol Vis Syst 2015; 2(3): 00046

[8] Jung JJ, Lim EH, Baek SH, et al. Attempts to Reduce the Progression of Myopia and Spectacle Prescriptions during ChildhoodL A Survey of Eye Specialists. Korean J Ophthalmol 2011; 25(6): 417-20.

[9] ECOO. The Ortkeratoloy Procedure. http://www.ecoo.info/wpcontent/uploads/2014/07/ECOO-Position-Paper-on-theOrthoK-Procedure_July-2014.pdf. (2014, accessed 23 February 2016).

[10] Charm J and Cho P. High Myopia - partial Reduction Orthokeratology (HM-PRO): Study design. Contact Lens and Anterior Eye. 2013; 36(4): 164-70.

[11] Chen C and Cho P. Toric Orthokeratology for High Myopic and Astigmatic Subjects for Myopic Control. Clin Exp Optom 2012; 95:1:103-8.

[12] Baertschi M and Wyss M. Correction of High Amounts of Astigmatism Through Orthokeratology. A Case Report. J Optom 2010; 3(4): 182-4.

[13] Si JK, Tang K, Bi HS, et al. Orthokeratology for Myopia Control: A Meta-analysis. Optometry and Vision Science 2015; 92(3): 252-7.

[14] Cho P, Cheung SW and Edwards M. The Longitudinal Orthokeratology Research in Children (LORIC) in Hong Kong: A Pilot Study on Refractive Changes and Myopic Control. Current Eye Research 2005; 30: 71-80.

[15] Chan B, Cho P and MPhil SWC. Orthokeratology Practice in Children in a University Clinic in Hong Kong. Clin Exp Optom 2008. 